\title{
Bayesian non-randomized response models for surveys with sensitive questions
}

\author{
Guo-Liang Tian, Kam Chuen Yuen, \\ Man-Lai TANg and Ming T. TAN
}

Three non-randomized response (NRR) models (namely, the non-randomized triangular, crosswise and hidden sensitivity models) have recently been developed for analyzing dichotomous sensitive questions. Unlike existing randomized response $(\mathrm{RR})$ models, no randomizing device is required for NRR models. This helps to reduce the cost, increase the efficiency, ensure the reproducibility, widen the applicability and encourage the cooperation. However, in applications (e.g., estimating the proportion of a rare sensitive attribute in a population) with highly skewed likelihood functions, classical asymptotic methods based on maximum likelihood estimates and their asymptotic standard errors may not be adequate. The purposes of this article are two folds. First, we develop Bayesian approaches for analyzing dichotomous sensitive questions based on the aforementioned NRR models. For both the non-randomized triangular and crosswise models, we obtain the exact posterior distribution and its explicit posterior moments, derive posterior mode via the EM algorithm and provide procedure for generating i.i.d. posterior samples. For the hidden sensitivity model, we consider Bayesian analysis under the commonly used conjugate Dirichlet prior. Second, noting that the covariance structure associated with the Dirichlet distribution is completely nonpositive, we propose three new joint priors for modeling independence structure with restrictions, negative correlation structure and positive correlation structure, respectively. A new hierarchical modeling strategy is provided. Importance sampling and data augmentation algorithm are employed to compute posterior moments and generate posterior samples. Three data sets from a sensitive sexual behavior study, an induced abortion study and a HIV study are used to illustrate the proposed methodologies.

KEYWORDS AND PHRASES: Bayesian method, DA algorithm, EM algorithm, Non-randomized response models, Randomized response technique, Sensitive questions.

\section{INTRODUCTION}

Asking people questions and collecting their responses is an important source of information that informs decision making in many medical studies, public health policies and

social issues. However, asking sensitive questions is generally seen as problematic in survey research due to concerns about information privacy. Because of these concerns, some respondents might intentionally give false information or simply refuse to divulge any information at all. As a result, directly asking sensitive questions is prone to error and bias. In order to increase the reliability and validity of responses, a number of strategies have been developed to minimize the likelihood of such error and bias.

The randomized response ( $\mathrm{RR}$ ) technique proposed by Warner (1965) is perhaps the first attempt to obtain more reliable information for estimating the proportion of a sensitive attribute in a population without revealing any respondent's actual status. However, a randomizing device (RD) is necessary and must be provided to each respondent to determine whether he/she needs to answer the sensitive question directly (with probability $p$ ) or the complement of the sensitive question (with probability $1-p$ ). Possible RDs include spinner with an arrow pointer, colored plastic balls/beads, coins, dice and poker chips. The requirement of RDs almost restricts the applicability of Warner RR model to face-toface interview only; otherwise, inevitably increases the cost of the survey. Even worse, Warner model does not work for $p=1 / 2$, which is a fatal limitation for obtaining trust from interviewees. In addition, Warner model is usually criticized by its inefficiency. To overcome some of the above limitations, Horvitz et al. (1967) and Greenberg et al. (1969) developed an unrelated question RR model. Subsequently, other authors (Kuk, 1990; Mangat \& Singh, 1990; Mangat, 1994; Chang \& Liang, 1996; Zou, 1997; Gjestvang \& Singh, 2006) suggested various modified RR models. Nonetheless, all these RR techniques heavily rely on those interviewercontrolled RDs, resulting in high cost, low cooperation and lack of reproductivity.

To overcome these drawbacks associated with RR models, two non-randomized response (NRR) models, namely the triangular and crosswise models, were developed recently by $\mathrm{Yu}$ et al. (2008) for a single sensitive dichotomous question. Tian et al. (2007) proposed a non-randomized hidden sensitivity (HS) model for analyzing the association between two sensitive dichotomous questions. Unlike traditional RR models, the NRR models utilize an independent (or unrelated) non-sensitive question (e.g., season of birth) in the survey to indirectly obtain a respondent's answer to a sensitive question. In general, the non-randomized triangular 
Table 1. The triangular model and the corresponding cell probabilities

\begin{tabular}{|c|c|c|c|c|c|}
\hline Categories & $W=0 W=1$ & Categories & $W=0$ & $W=1$ & Total \\
\hline$Y=0$ & $\bigcirc \quad \bullet$ & $Y=0$ & $(1-\pi)(1-p)$ & $(1-\pi) p$ & $1-\pi$ \\
\hline$Y=1$ & $\bullet$ & $Y=1$ & $\pi(1-p)$ & $\pi p$ & $\pi$ \\
\hline & & Total & $1-p$ & $p$ & 1 \\
\hline
\end{tabular}

Respondent: Please truthfully put a tick in the circle or in the triangle formed by the three dots.

design is more efficient than the randomized Warner design. Most importantly, all NRR designs do not require any RDs and hence substantially reduce the cost, improve the cooperation, and ensure the reproductivity.

Although the aforementioned NRR models have been shown to be very useful to surveys involving sensitive questions, existing classical analysis methods based on maximum likelihood estimates and their asymptotic standard errors may not be adequate in applications (e.g., estimating the proportion of a rare sensitive attribute in a population) with highly skewed likelihood function. ${ }^{1}$ In addition, when investigators have some knowledge about the parameters of interest before they obtain the data, Bayesian estimation methods may be more appealing. For instance, Greenberg et al. (1969) suggested that if a membership in the sensitive group really possesses a socially disapproved attribute it is reasonable to assume that the corresponding proportion should be in neighborhood of 0.05 and 0.10 . Winkler \& Frankin (1979), Pitz (1980) and Spurrier \& Padgett (1980) presented Bayesian approaches for the Warner and unrelated question RR models, respectively, using parametric models of prior information. Using the Bayes linear estimator, similar results were obtained by O'Hagan (1987) from a nonparametric model. Migon \& Tachibana (1997) considered Bayesian approximation in RR model. Using the Gibbs sampler, Unnikrisknan \& Kunte (1999) developed an unified model for RR strategies of which the Warner, unrelated question $\mathrm{RR}$ as well as polychotomous models are special cases. Bar-Lev et al. (2003) presented a common Bayesian approach to four RR models. DiPietro (2004) described a data analysis project in the Bayesian framework. Kim et al. (2006) provided Bayesian methods for Mangat's (1994) RR model.

The purposes of this article are two-fold. First, we develop Bayesian approaches for the aforementioned three NRR models under the commonly used beta or Dirichlet priors. The main advantage of adopting the conjugate prior is for its mathematical and computational simplicity. It is well known that the covariance structure associated with the Dirichlet distribution is completely non-positive. For those cases that require positive covariance structures, the Dirichlet distribution is not appropriate. Hence, it is the second purpose of this paper to propose three new priors for modeling independence structure with restrictions, negative correlation

\footnotetext{
${ }^{1}$ See Figure $1(\mathrm{~b})$ and (c) in $\S 3$. We note that the likelihood function is entirely identical to the posterior distribution when the prior is a uniform distribution.
}

structure and positive correlation structure, respectively. A new hierarchical modeling strategy is also proposed.

The rest of the paper are organized as follows. We first briefly review the three NRR models and discuss some of their advantages over the RR models in Section 2. In Sections 3 and 4, we obtain the exact posterior distributions and their explicit posterior moments, derive posterior modes via the EM algorithm and provide approach to generate i.i.d. posterior samples for both the triangular and crosswise models, respectively. In Section 5, we present the Bayesian analysis for the HS model under a conjugate Dirichlet prior. We then propose three new priors for different covariance structures. Importance sampling and data augmentation (DA) algorithms are employed to compute the posterior moments and generate posterior samples under the positive covariance structure. Three data sets from a sensitive sexual behavior study, an induced abortion study and a HIV study are used to illustrate the proposed methods in Section 6. We finally conclude in Section 7.

\section{NON-RANDOMIZED RESPONSE MODELS}

\subsection{The non-randomized triangular model for one sensitive question}

Let $Y=1$ denote the class of people with a sensitive characteristic (e.g., drug-taking) and $Y=0$ the complementary class. Let $W$ be a dichotomous variate associated with a non-sensitive question and independent of $Y$. For instance, $W=1$ may represent the class of people who were born between July and December and $W=0$ represents the corresponding complementary class. The survey designer should choose an appropriate $W$ in such a way that the proportion $p=\operatorname{Pr}(W=1)$ is either known or can be estimated easily. Without loss of generality, let $p$ be known. The purpose is to estimate the proportion $\pi=\operatorname{Pr}(Y=1)$.

For a face-to-face personal interview, the survey designer may replace the sensitive question by the tabular form presented on the left-hand side of Table 1 and ask the respondent to put a tick in the circle or in the triangle formed by the three dots according to his/her truthful status. It is noteworthy that respondents to $\{Y=0, W=0\}$ are simply nondrug users born between January and June. In other words, $\{Y=0, W=0\}$ represents a non-sensitive subclass. On the other hand, a tick in the triangle indicates the respondent can be either a drug user or a non-drug user born between 
Table 2. The crosswise model (Yu et al., 2008)

\begin{tabular}{|c|c|c|c|c|c|}
\hline Categories & $W=0 W=1$ & Categories & $W=0$ & $W=1$ & Total \\
\hline$Y=0$ & $\bigcirc \bullet$ & $Y=0$ & $(1-\pi)(1-p)$ & $(1-\pi) p$ & $1-\pi$ \\
\hline$Y=1$ & $\bigcirc$ & $Y=1$ & $\pi(1-p)$ & $\pi p$ & $\pi$ \\
\hline & & Total & $1-p$ & $p$ & 1 \\
\hline
\end{tabular}

Respondent: Please truthfully put a tick in the diagonal with the two circles or the off-diagonal with the two dots.

Table 3. Questionnaire for the hidden sensitivity (HS) model

\begin{tabular}{l|c|c|c|c}
\hline \hline Categories & $W=1$ & $W=2$ & $W=3$ & $W=4$ \\
\hline I: $\{X=0, Y=0\}$ & Block 1: & Block 2: & Block 3: & Block 4: \\
\hline II: $\{X=0, Y=1\}$ & \multicolumn{3}{c}{ Category II: please put a tick in Block 2 } \\
\hline III: $\{X=1, Y=0\}$ & \multicolumn{3}{c}{ Category III: please put a tick in Block 3 } \\
\hline IV $:\{X=1, Y=1\}$ & \multicolumn{3}{r}{ Category IV: please put a tick in Block 4 } \\
\hline
\end{tabular}

July and December. Therefore, $\{Y=1\} \cup\{Y=0, W=1\}$ can be regarded as a non-sensitive subclass as well. Such camouflage would presumably encourage respondents to not only participate in the survey but also provide truthful responses. In all subsequent discussion, this is called the triangular model.

\subsection{The crosswise model for one sensitive question}

Besides the triangular model, we can consider the following so-called crosswise model for analyzing a single sensitive question. Let $Y$ and $W$ be defined in $\S 2.1, p=\operatorname{Pr}(W=1)$ and $\pi=\operatorname{Pr}(Y=1)$. The interviewer may reformulate the sensitive question in the format as shown on the lefthand side of Table 2 and ask the interviewee to truthfully put a tick in either the diagonal with two circles or the off-diagonal with two dots. It is important to notice that both $\{Y=0, W=0\} \cup\{Y=1, W=1\}$ and $\{Y=0, W=1\} \cup\{Y=1, W=0\}$ are non-sensitive subclasses. Thus, whether an interviewee possesses the sensitive attribute will not be exposed. Yu et al. (2008) showed that this crosswise model is a non-randomized version of the original Warner model.

\subsection{The hidden sensitivity model for two sensitive questions}

Consider two binary sensitive variates $X$ and $Y$. For the sensitive variate $X$, let $X=1$ denote the sensitive attribute of a respondent (e.g., taking drug), and $X=0$ the nonsensitive one (e.g., not taking drug). For the sensitive variate $Y$, let $Y=1$ be the other sensitive attribute (e.g., HIV+) and $Y=0$ the non-sensitive one (e.g., HIV-). Let $\theta_{x}=$ $\operatorname{Pr}(X=1), \theta_{y}=\operatorname{Pr}(Y=1), \theta_{1}=\operatorname{Pr}(X=0, Y=0)$, $\theta_{2}=\operatorname{Pr}(X=0, Y=1), \theta_{3}=\operatorname{Pr}(X=1, Y=0)$ and $\theta_{4}=$ $\operatorname{Pr}(X=1, Y=1)$. Hence, $\theta_{x}=\theta_{3}+\theta_{4}$ and $\theta_{y}=\theta_{2}+\theta_{4}$. A commonly used measure of association is the odds ratio defined as $\delta=\theta_{1} \theta_{4} /\left(\theta_{2} \theta_{3}\right)$. The objective is to estimate $\theta_{x}$, $\theta_{y}, \theta_{i} \mathrm{~s}$ and $\delta$.

To obtain reliable responses from respondents, we introduce a non-sensitive variate $W$, which is independent of $(X, Y)$, with four mutually exclusive categories. Let $p_{i}=$ $\operatorname{Pr}(W=i)$ for $i=1, \ldots, 4$. Like the triangular and crosswise models, the variate $W$ should be chosen in such a way that all $p_{i}$ s can be obtained or estimated easily. Therefore, we assume that $p_{i} \mathrm{~s}$ are known. For example, let $\{W=i\}$ denote that a respondent was born in the $i$-th quarter and we can thus assume that $p_{i}$ s are approximately all equal to $1 / 4$.

Instead of directly answering the sensitive question, each respondent is asked to answer a new question as shown in Table 3. Since $\{X=0, Y=0\}$ represents an non-sensitive subclass, we have reason to believe that a respondent will put a tick in Block $i(i=1, \ldots, 4)$ according to his/her truthful status if (s)he belongs to this category. The other categories (i.e., Blocks II to IV), however, are sensitive to the respondent. If the respondent belongs to Block II (III or IV), (s)he is requested/forced to put a tick in Block 2 (3 or 4) so that his/her privacy is somehow protected. This technique is simply called the hidden sensitivity model in the sense that the sensitive attribute of a respondent is being hidden. Table 4 shows the cell probabilities $\theta_{i}$ s and the observed frequencies $n_{i}$ s. Let $n_{1}$ denote the observed frequency of respondents putting a tick in Block 1. $n_{2}$ represents the sum of the frequencies of respondents belonging to Block 2 and Block II. We can interpret $n_{3}$ and $n_{4}$ similarly.

Unlike the popular randomized response models, it is noteworthy that all the aforementioned NRR models have the following advantages: (i) they do not require any RDs and the study cost is thus reduced; (ii) the results can be reproducible; (iii) they can be easily operated for both interviewers and interviewees; and (iv) they can be applied to both face-to-face personal interviews and mail questionnaires. 
Table 4. Cell probabilities, observed and unobservable frequencies for the HS model

\begin{tabular}{|c|c|c|c|c|c|}
\hline Categories & $W=1$ & $W=2$ & $W=3$ & $W=4$ & Total \\
\hline I: $\{X=0, Y=0\}$ & $p_{1} \theta_{1}$ & $p_{2} \theta_{1}$ & $p_{3} \theta_{1}$ & $p_{4} \theta_{1}$ & $\theta_{1}\left(Z_{1}\right)$ \\
\hline II: $\{X=0, Y=1\}$ & & & & & $\theta_{2}\left(Z_{2}\right)$ \\
\hline III: $\{X=1, Y=0\}$ & & & & & $\theta_{3}\left(Z_{3}\right)$ \\
\hline $\mathrm{IV}:\{X=1, Y=1\}$ & & & & & $\theta_{4}\left(Z_{4}\right)$ \\
\hline Total & $p_{1}\left(n_{1}\right)$ & $p_{2}\left(n_{2}\right)$ & $p_{3}\left(n_{3}\right)$ & $p_{4}\left(n_{4}\right)$ & $1(n)$ \\
\hline
\end{tabular}

Note: $n=\sum_{i=1}^{4} n_{i}, Z_{1}=n-\left(Z_{2}+Z_{3}+Z_{4}\right)$, where $\left(Z_{2}, Z_{3}, Z_{4}\right)$ are unobservable.

(a) $n=200, s=135$

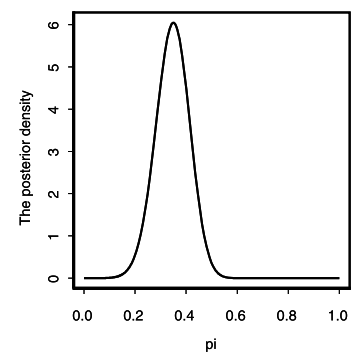

(b) $n=200, s=115$

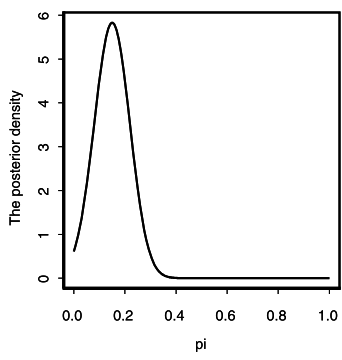

(c) $n=200, s=105$

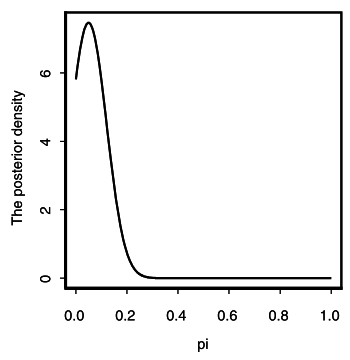

Figure 1. Posterior distributions for $\pi$ under the uniform prior (i.e., $a=b=1)$ and $p=\operatorname{Pr}(W=1)=0.5$ for the non-randomized triangular model. (a) $n=200$ and $s=135$; (b) $n=200$ and $s=115$; (c) $n=200$ and $s=105$.

\section{BAYESIAN METHODS FOR THE NON-RANDOMIZED TRIANGULAR MODEL}

In this section, we first derive the exact posterior distribution of $\pi$ and its explicit posterior moments. We then derive the posterior mode via the EM algorithm (Dempster et al., 1977) when the posterior distribution of $\pi$ is highly skewed. Finally, we utilize the exact inverse Bayes formulae (IBF) sampler (Tian, Tan \& Ng, 2007) to generate i.i.d. posterior samples.

\subsection{Posterior moments in closed-form}

For the triangular model given in Table 1, we define a 'hidden' variable $Y^{\mathrm{HT}}$ as follows:

$$
Y^{\mathrm{HT}}=\left\{\begin{array}{c}
1, \quad \text { with probability } \pi+(1-\pi) p, \\
\text { if a tick is put in the triangle } \\
0, \quad \text { with probability }(1-\pi)(1-p), \\
\text { if a tick is put in the circle }
\end{array}\right.
$$

Let $Y_{\mathrm{obs}}=\left\{y_{i}^{\mathrm{HT}}: i=1, \ldots, n\right\}$ denote the observed data for the $n$ respondents with $y_{i}^{\mathrm{HT}}=1$ if the $i$-th respondent puts a tick in the triangle; $=0$ otherwise. The likelihood function for $\pi$ is then given by

$$
\begin{aligned}
L_{T}\left(\pi \mid Y_{\mathrm{obs}}\right) & =\prod_{i=1}^{n}[\pi+(1-\pi) p]^{y_{i}^{\mathrm{HT}}}[(1-\pi)(1-p)]^{1-y_{i}^{\mathrm{HT}}} \\
& =[\pi+(1-\pi) p]^{s}[(1-\pi)(1-p)]^{n-s}, 0 \leq \pi \leq 1,
\end{aligned}
$$

where $s \hat{=} \sum_{i=1}^{n} y_{i}^{\mathrm{HT}}$. If we choose the beta distribution $\operatorname{Beta}(a, b)$ to be the prior distribution of $\pi$, then the posterior distribution of $\pi$ takes the following closed-form expression:

$$
\begin{aligned}
f\left(\pi \mid Y_{\mathrm{obs}}\right)= & c_{T}^{-1}(a, b ; s, n-s) \\
& \times \pi^{a-1}(1-\pi)^{b+n-s-1}[\pi+(1-\pi) p]^{s},
\end{aligned}
$$

where the normalizing constant is given by

$$
c_{T}(a, b ; s, n-s)=\sum_{j=0}^{s}\left(\begin{array}{l}
s \\
j
\end{array}\right) p^{s-j} B(a+j, b+n-j) .
$$

For $a=b=1$ and $p=\operatorname{Pr}(W=1)=0.5$, Figure 1 shows the posterior distributions of $\pi$ for three different combinations of $n$ and $s$.

When $f\left(\pi \mid Y_{\text {obs }}\right)$ is fairly symmetric, the first two posterior moments are good enough to describe the location and discrepancy of the posterior distribution. From (3.1), the $t$-th posterior moment of $\pi$ has the following explicit expression:

$$
E\left(\pi^{t} \mid Y_{\mathrm{obs}}\right)=\frac{c_{T}(a+t, b ; s, n-s)}{c_{T}(a, b ; s, n-s)}, \quad t \geq 1
$$

\subsection{Calculation of the posterior mode via the EM algorithm}

When $f\left(\pi \mid Y_{\text {obs }}\right)$ is highly skewed (for instance, see Figure $1(\mathrm{c})$ ), the posterior mode is usually adopted for describing the location. To derive the mode, we first introduce an unobservable variable $Z$, which denotes the number of respondents with the sensitive attribute. Obviously, 
the number of respondents without the sensitive characteristic is $n-Z$. Thus, the complete-data is $Y_{\text {com }}=\left\{Y_{\text {obs }}, Z\right\}$. The complete-data posterior distribution and the conditional predictive distribution are given by

$$
f\left(\pi \mid Y_{\mathrm{obs}}, Z\right)=\operatorname{Beta}(\pi \mid a+Z, b+n-Z),
$$

and

$$
f\left(Z \mid Y_{\mathrm{obs}}, \pi\right)=\operatorname{Binomial}(Z \mid s, \pi /[\pi+(1-\pi) p])
$$

respectively. Using the EM algorithm, the M-step computes the complete-data posterior mode as

$$
\tilde{\pi}_{T}=\frac{a+Z-1}{a+b+n-2}
$$

and the E-step is to replace $Z$ by its conditional expectation

$$
E\left(Z \mid Y_{\mathrm{obs}}, \pi\right)=\frac{s \pi}{\pi+(1-\pi) p} .
$$

\subsection{Generation of i.i.d. posterior samples via the exact IBF sampling}

We re-write (3.4) and (3.5) as $f\left(\pi \mid Y_{\text {obs }}, Z\right)=\operatorname{Beta}(\pi \mid a+$ $Z, b+n-Z)$ and $f\left(Z \mid Y_{\mathrm{obs}}, \pi\right)=\operatorname{Binomial}(Z \mid s, \pi /[\pi+(1-$ $\pi) p]$ ). According to the exact IBF algorithm presented in Appendix A, to generate i.i.d. posterior samples we simply need to identify $\mathcal{S}_{\left(Z \mid Y_{\text {obs }}\right)}$ and calculate $\left\{\omega_{k}\right\}_{k=1}^{K}$. Obviously,

$$
\mathcal{S}_{\left(Z \mid Y_{\mathrm{obs}}\right)}=\mathcal{S}_{\left(Z \mid Y_{\mathrm{obs}}, \pi\right)}=\left\{z_{1}, \ldots, z_{K}\right\}=\{0,1, \ldots, s\}
$$

and $K=s+1$. Setting $\pi_{0}=0.5$, from (A.2) and (A.3), we obtain

$$
\begin{aligned}
q_{k}(0.5) & =\frac{\left(\begin{array}{c}
s \\
z_{k}
\end{array}\right) p^{s-z_{k}} /(1+p)^{s}}{0.5^{a+b+n} / B\left(a+z_{k}, b+n-z_{k}\right)} \\
& \propto\left(\begin{array}{c}
s \\
z_{k}
\end{array}\right) \frac{\Gamma\left(a+z_{k}\right) \Gamma\left(b+n-z_{k}\right)}{p^{z_{k}}},
\end{aligned}
$$

for $k=1, \ldots, K$, and $\omega_{k}=q_{k}(0.5) / \sum_{k^{\prime}=1}^{K} q_{k^{\prime}}(0.5)$.

\section{BAYESIAN METHODS FOR THE CROSSWISE MODEL}

\subsection{Posterior moments in closed-form}

Let $Y_{\mathrm{obs}}=\left\{y_{i}^{\mathrm{HW}}: i=1, \ldots, n\right\}$ denote the observed data for the $n$ respondents, where $y_{i}^{\mathrm{HW}}=1$ if the $i$-th respondent puts a tick in the main diagonal with the two circles; $=0$ otherwise. The likelihood function for $\pi$ is then given by

$$
\begin{aligned}
L_{W}\left(\pi \mid Y_{\mathrm{obs}}\right)= & \prod_{i=1}^{n}[\pi p+(1-\pi)(1-p)]^{y_{i}^{\mathrm{HW}}} \\
& \cdot[\pi(1-p)+(1-\pi) p]^{1-y_{i}^{\mathrm{HW}}} \\
= & {[\pi p+(1-\pi)(1-p)]^{r} } \\
& \cdot[\pi(1-p)+(1-\pi) p]^{n-r}, \quad 0 \leq \pi \leq 1,
\end{aligned}
$$

where $r \hat{=} \sum_{i=1}^{n} y_{i}^{\mathrm{HW}}$. Assume that $\pi \sim \operatorname{Beta}(a, b)$. Thus, the posterior distribution of $\pi$ is

$$
f\left(\pi \mid Y_{\mathrm{obs}}\right)=\frac{\pi^{a-1}(1-\pi)^{b-1} L_{W}\left(\pi \mid Y_{\mathrm{obs}}\right)}{c_{W}(a, b ; r, n-r)},
$$

where the normalizing constant $c_{W}(a, b ; r, n-r) \hat{=}$ $\frac{p^{n-r}(1-p)^{r}}{\Gamma(a+b+n)} c_{W}^{*}(a, b ; r, n-r)$ and

$$
\begin{aligned}
c_{W}^{*}(a, b ; r, n-r)= & \sum_{j_{1}=0}^{r} \sum_{j_{2}=0}^{n-r}\left(\begin{array}{c}
r \\
j_{1}
\end{array}\right)\left(\begin{array}{c}
n-r \\
j_{2}
\end{array}\right)\left(\frac{p}{1-p}\right)^{j_{1}-j_{2}} \\
& \cdot \Gamma\left(a+j_{1}+j_{2}\right) \Gamma\left(b+n-j_{1}-j_{2}\right) .
\end{aligned}
$$

Therefore, the $t$-th posterior moment of $\pi$ is given by

$$
E\left(\pi^{t} \mid \text { data }\right)=\frac{c_{W}^{*}(a+t, b ; r, n-r)}{c_{W}^{*}(a, b ; r, n-r)} \cdot \frac{\Gamma(a+b+n)}{\Gamma(a+t+b+n)}, t \geq 1 .
$$

\subsection{Calculation of the posterior mode via the EM algorithm}

To derive the posterior mode, we first introduce two unobservable variables $Z=\left(Z_{1}, Z_{2}\right)$, where $Z_{1}$ and $Z_{2}$ are respectively the counts of cell- $(1,1)$ and cell- $(1,0)$ in Table 2. Thus, the complete-data posterior distribution and the conditional predictive distribution are given by

$f\left(\pi \mid Y_{\text {obs }}, Z\right)=\operatorname{Beta}\left(\pi \mid a+Z_{1}+Z_{2}, b+n-Z_{1}-Z_{2}\right), \quad$ and

$$
\begin{aligned}
f\left(Z \mid Y_{\mathrm{obs}}, \pi\right)= & f\left(Z_{1} \mid Y_{\mathrm{obs}}, \pi\right) \cdot f\left(Z_{2} \mid Y_{\mathrm{obs}}, \pi\right) \\
= & \text { Binomial }\left(Z_{1} \mid r, \frac{\pi p}{\pi p+(1-\pi)(1-p)}\right) \\
& \cdot \text { Binomial }\left(Z_{2} \mid n-r, \frac{\pi(1-p)}{\pi(1-p)+(1-\pi) p}\right),
\end{aligned}
$$

respectively. Using the EM algorithm, the M-step yields the following complete-data posterior mode

$$
\tilde{\pi}_{W}=\frac{a+Z_{1}+Z_{2}-1}{a+b+n-2}
$$

while the E-step is to replace $Z_{1}+Z_{2}$ by its conditional expectation

$$
\begin{aligned}
E\left(Z_{1}+Z_{2} \mid Y_{\mathrm{obs}}, \pi\right)= & \frac{r \pi p}{\pi p+(1-\pi)(1-p)} \\
& +\frac{(n-r) \pi(1-p)}{\pi(1-p)+(1-\pi) p}, \quad p \neq 1 / 2 .
\end{aligned}
$$

In fact, when $p=1 / 2$, (4.6) becomes $E\left(Z_{1}+Z_{2} \mid Y_{\text {obs }}, \pi\right)=$ $n \pi$, which does not depend on the observed data $Y_{\text {obs }}$ nor $r$. 
In this case, the EM algorithm in (4.5) and (4.6) converges in one step and we have $\tilde{\pi}_{W}=(a-1) /(a+b-2)$, which is actually the mode of the prior distribution $\operatorname{Beta}(a, b)$.

\subsection{Generation of i.i.d. posterior samples via the exact IBF sampling}

To apply the exact IBF algorithm to the present model, we simply need to identify the conditional support of $Z \mid\left(Y_{\text {obs }}, \pi\right)$. From (4.4), we have

$$
\begin{aligned}
\mathcal{S}_{\left(Z \mid Y_{\mathrm{obs}}\right)} & \mathcal{S}_{\left(Z \mid Y_{\mathrm{obs}}, \pi\right)}=\left\{z_{1}, \ldots, z_{K}\right\} \\
& =\left\{\begin{array}{cccc}
(0,0) & (0,1) & \cdots & (0, n-r) \\
(1,0) & (1,1) & \cdots & (1, n-r) \\
\vdots & \vdots & \ddots & \vdots \\
(r, 0) & (r, 1) & \cdots & (r, n-r)
\end{array}\right\},
\end{aligned}
$$

where $K=(r+1)(n-r+1)$. We then calculate $\left\{\omega_{k}\right\}_{k=1}^{K}$ according to (A.2) and (A.3) with $\pi_{0}=0.5$.

\section{BAYESIAN METHODS FOR THE HIDDEN SENSITIVITY MODEL}

\subsection{Bayesian inferences under Dirichlet prior}

Assume that there are totally $n$ respondents with $n_{i}$ ticks being put in Block $i$ for $i=1, \ldots, 4$ (see Table 4$)$. Let $Y_{\mathrm{obs}}=$ $\left\{n ; n_{1}, \ldots, n_{4}\right\}$ denote the observed frequencies with $n=$ $\sum_{i=1}^{4} n_{i}$ and $\boldsymbol{\theta}=\left(\theta_{1}, \ldots, \theta_{4}\right)^{\top} \in \mathbb{T}_{4}$ be the cell probability vector, where $\mathbb{T}_{4} \hat{=}\left\{\left(\theta_{1}, \ldots, \theta_{4}\right)^{\top}: \theta_{i} \geq 0, \sum_{i=1}^{4} \theta_{i}=1\right\}$. The observed-data likelihood function for $\boldsymbol{\theta}$ is then

$$
L_{H}\left(\boldsymbol{\theta} \mid Y_{\text {obs }}\right)=\theta_{1}^{n_{1}} \prod_{i=2}^{4}\left(p_{i} \theta_{1}+\theta_{i}\right)^{n_{i}}, \quad \boldsymbol{\theta} \in \mathbb{T}_{4},
$$

where $p_{i}=\operatorname{Pr}(W=i), i=1, \ldots, 4$, are assumed to be known constants.

\subsubsection{Posterior moments in closed-form}

The natural prior for $\boldsymbol{\theta}$ is the Dirichlet distribution $\operatorname{Dirichlet}(\mathbf{a})$ with $\mathbf{a}=\left(a_{1}, \ldots, a_{4}\right)^{\top}$. Thus, the posterior distribution of $\boldsymbol{\theta}$ has the following closed-form expression:

$$
f\left(\boldsymbol{\theta} \mid Y_{\mathrm{obs}}\right)=c_{H}^{-1}(\mathbf{a}, \mathbf{n}) \cdot \prod_{i=1}^{4} \theta_{k}^{a_{i}-1} \cdot L_{H}\left(\boldsymbol{\theta} \mid Y_{\mathrm{obs}}\right), \quad \boldsymbol{\theta} \in \mathbb{T}_{4}
$$

where the normalizing constant $c_{H}(\mathbf{a}, \mathbf{n})=$ $c_{H}^{*}(\mathbf{a}, \mathbf{n}) / \Gamma\left(\sum_{i=1}^{4} a_{i}+n\right)$ and

$$
\begin{aligned}
c_{H}^{*}(\mathbf{a}, \mathbf{n})= & \sum_{j_{2}=0}^{n_{2}} \sum_{j_{3}=0}^{n_{3}} \sum_{j_{4}=0}^{n_{4}}\left\{\Gamma\left(a_{1}+n_{1}+j_{2}+j_{3}+j_{4}\right)\right. \\
& \left.\cdot \prod_{\ell=2}^{4}\left(\begin{array}{c}
n_{\ell} \\
j_{\ell}
\end{array}\right) \Gamma\left(a_{\ell}+n_{\ell}-j_{\ell}\right) p_{\ell}^{j_{\ell}}\right\} .
\end{aligned}
$$

The posterior moment of $\boldsymbol{\theta}$ is given by

$E\left(\theta_{1}^{t_{1}} \theta_{2}^{t_{2}} \theta_{3}^{t_{3}} \theta_{4}^{t_{4}} \mid Y_{\mathrm{obs}}\right)=\frac{c_{H}^{*}(\mathbf{a}+\mathbf{t}, \mathbf{n})}{c_{H}^{*}(\mathbf{a}, \mathbf{n})} \cdot \frac{\Gamma\left(\sum_{i=1}^{4} a_{i}+n\right)}{\Gamma\left(\sum_{i=1}^{4}\left(a_{i}+t_{i}\right)+n\right)}$.

Hence, the posterior moments of $\theta_{x}, \theta_{y}$ and $\delta$ can be readily expressed as

$$
\begin{aligned}
E\left(\theta_{x} \mid Y_{\text {obs }}\right) & =E\left(\theta_{3} \mid Y_{\text {obs }}\right)+E\left(\theta_{4} \mid Y_{\text {obs }}\right) \\
E\left(\theta_{x}^{2} \mid Y_{\text {obs }}\right) & =E\left(\theta_{3}^{2} \mid Y_{\text {obs }}\right)+2 E\left(\theta_{3} \theta_{4} \mid Y_{\text {obs }}\right)+E\left(\theta_{4}^{2} \mid Y_{\text {obs }}\right) \\
E\left(\theta_{y} \mid Y_{\text {obs }}\right) & =E\left(\theta_{2} \mid Y_{\text {obs }}\right)+E\left(\theta_{4} \mid Y_{\text {obs }}\right) \\
E\left(\theta_{y}^{2} \mid Y_{\text {obs }}\right) & =E\left(\theta_{2}^{2} \mid Y_{\text {obs }}\right)+2 E\left(\theta_{2} \theta_{4} \mid Y_{\text {obs }}\right)+E\left(\theta_{4}^{2} \mid Y_{\text {obs }}\right) \\
E\left(\delta \mid Y_{\text {obs }}\right) & =E\left(\theta_{1} \theta_{2}^{-1} \theta_{3}^{-1} \theta_{4} \mid Y_{\text {obs }}\right), \quad \text { and } \\
E\left(\delta^{2} \mid Y_{\text {obs }}\right) & =E\left(\theta_{1}^{2} \theta_{2}^{-2} \theta_{3}^{-2} \theta_{4}^{2} \mid Y_{\text {obs }}\right)
\end{aligned}
$$

5.1.2. Calculation of the posterior mode via the EM algorithm

To derive the posterior mode of $\boldsymbol{\theta}$, we treat the observed frequencies $n_{2}, n_{3}$ and $n_{4}$ as incomplete data and the frequencies $Z_{2}, Z_{3}$ and $Z_{4}$ as missing data (see Table 4). Let $Z=\left(Z_{2}, Z_{3}, Z_{4}\right)^{\top}$ with $Z_{1}=n-Z_{2}-Z_{3}-Z_{4}$. Thus, the complete-data posterior distribution and the conditional predictive distribution are given by

$$
f\left(\boldsymbol{\theta} \mid Y_{\mathrm{obs}}, Z\right)=\operatorname{Dirichlet}\left(\boldsymbol{\theta} \mid a_{1}+Z_{1}, \ldots, a_{4}+Z_{4}\right),
$$

and

$$
f\left(Z \mid Y_{\mathrm{obs}}, \boldsymbol{\theta}\right)=\prod_{i=2}^{4} \operatorname{Binomial}\left(Z_{i} \mid n_{i}, \theta_{i} /\left[p_{i} \theta_{1}+\theta_{i}\right]\right),
$$

respectively. Based on the EM algorithm, the M-step calculates the complete-data posterior mode by

$$
\tilde{\theta}_{i}=\frac{a_{i}+Z_{i}-1}{\sum_{\ell=1}^{4} a_{\ell}+n-4}, \quad i=2,3,4, \tilde{\theta}_{1}=1-\tilde{\theta}_{2}-\tilde{\theta}_{3}-\tilde{\theta}_{4},
$$

and the E-step is to replace $\left\{Z_{i}\right\}$ by their conditional expectations

$$
E\left(Z_{i} \mid Y_{\mathrm{obs}}, \boldsymbol{\theta}\right)=\frac{n_{i} \theta_{i}}{p_{i} \theta_{1}+\theta_{i}}, \quad i=2,3,4
$$

5.1.3. Generation of posterior samples via the DA algorithm

Based on (5.3) and (5.4), we can use the DA algorithm (Tanner \& Wong, 1987) to generate posterior samples of $\boldsymbol{\theta}$. We may choose $\boldsymbol{\theta}_{0}=(0.25, \ldots, 0.25)^{\top}$ as the initial value.

\subsection{Bayesian inferences under other priors}

In the previous section, we consider the Dirichlet distribution as the prior of $\boldsymbol{\theta}$. It is well known that the covariance structure associated with the Dirichlet distribution is completely non-positive. Obviously, those cases that possess, for instance, positive covariance structures cannot be modeled 
Table 5. Parameter spaces $\Theta$ and $\Theta_{x}$ and their cell probabilities

\begin{tabular}{c|cc|cc|ccc|c}
\hline \hline Categories & $Y=0$ & $Y=1$ & Marginal & & Categories & $Y=0$ & $Y=1$ & Marginal \\
\hline$X=0$ & $\theta_{1}$ & $\theta_{2}$ & $\theta_{1}+\theta_{2}$ & & $X=0$ & $\left(1-\theta_{x}\right) \xi$ & $\left(1-\theta_{x}\right)(1-\xi)$ & $1-\theta_{x}$ \\
$X=1$ & $\theta_{3}$ & $\theta_{4}$ & $\theta_{3}+\theta_{4}$ & & $X=1$ & $\theta_{x}(1-\eta)$ & $\theta_{x} \eta$ & $\theta_{x}$ \\
\cline { 1 - 3 } & & & 1 & & Space $\Theta_{x}$ & & & 1 \\
\hline
\end{tabular}

by the Dirichlet prior. To explore the essence of the Dirichlet prior, we first transform the original parameter space $\Theta=\left\{\theta_{1}, \theta_{2}, \theta_{3}\right\}$ into an orthogonal parameter space, say $\Theta_{x}=\left\{\theta_{x}, \xi, \eta\right\}$, and present an equivalent prior distribution for $\left(\theta_{x}, \xi, \eta\right)^{\top}$ when $\left(\theta_{1}, \theta_{2}, \theta_{3}\right)^{\top}$ follows a Dirichlet distribution. Next, we develop three new joint priors for $\left(\theta_{x}, \xi, \eta\right)^{\top}$ for modeling (i) independence structure with restrictions, (ii) negative correlation structure, and (iii) positive correlation structures. Finally, for the positive correlation structure, we derive the corresponding posterior moments for the parameters of interest via the importance sampling and generate posterior samples via the DA algorithm.

\subsubsection{Orthogonal parameter space}

Let $\theta_{x}=\operatorname{Pr}(X=1)$ denote the marginal probability of $X, \xi=\operatorname{Pr}(Y=0 \mid X=0)$ and $\eta=\operatorname{Pr}(Y=1 \mid X=1)$ be the corresponding conditional probabilities. Table 5 illustrates the fundamental relationship between the two parameter spaces $\Theta=\left\{\theta_{1}, \theta_{2}, \theta_{3}\right\}$ and $\Theta_{x}=\left\{\theta_{x}, \xi, \eta\right\}$.

It is noteworthy that the following one-to-one transformation

$$
\theta_{x}=\theta_{3}+\theta_{4}, \quad \xi=\frac{\theta_{1}}{\theta_{1}+\theta_{2}}, \quad \text { and } \quad \eta=\frac{1-\theta_{1}-\theta_{2}-\theta_{3}}{1-\theta_{1}-\theta_{2}}
$$

maps the original parameter space $\Theta$ into the orthogonal parameter space (i.e., an unit cube in $\mathbb{R}^{3}$ ) $\Theta_{x}$. The corresponding Jacobian is given by

$$
|J|=\left|\frac{\partial\left(\theta_{1}, \theta_{2}, \theta_{3}\right)}{\partial\left(\theta_{x}, \xi, \eta\right)}\right|=\theta_{x}\left(1-\theta_{x}\right) .
$$

We have the following result.

Theorem 1. If $\left(\theta_{1}, \theta_{2}, \theta_{3}\right)^{\top}$ follows the Dirichlet prior $\operatorname{Dirichlet}\left(a_{1}, a_{2}, a_{3} ; a_{4}\right)$ in space $\Theta$, the equivalent prior distribution of $\left(\theta_{x}, \xi, \eta\right)^{\top}$ in $\Theta_{x}$ is given by

$$
\begin{aligned}
\theta_{x} & \sim \operatorname{Beta}\left(a_{3}+a_{4}, a_{1}+a_{2}\right), \\
\xi & \sim \operatorname{Beta}\left(a_{1}, a_{2}\right), \quad \text { and } \\
\eta & \sim \operatorname{Beta}\left(a_{4}, a_{3}\right), \quad \text { or } 1-\eta \sim \operatorname{Beta}\left(a_{3}, a_{4}\right),
\end{aligned}
$$

where $\theta_{x}, \xi$ and $\eta$ are mutually independent.

\subsubsection{Joint prior for modeling independence with restric- tions}

Sometimes, prior information on $\theta_{x}, \xi$ and $\eta$ are available in the form of restrictions. For example, let $X=1$ if a person has annual income being greater than or equal to $\$ 100,000$; $=0$ otherwise, and $Y=1$ if a person travels at least once every year; $=0$ otherwise. Thus, we have $\eta=\operatorname{Pr}$ (a person travels once every year| the annual income $\geq \$ 100,000)$ and $\xi=\operatorname{Pr}$ (a person does not travel at all every year| the annual income $\geq \$ 100,000)$. In general, the possibility of traveling every year is positively related to annual income. Therefore, it is reasonable to impose the following restrictions on $\eta$ and $\xi$,

$$
\eta \geq 1-\eta, \quad \text { and } \quad \xi \geq 1-\xi
$$

i.e., $\eta \geq 0.5, \xi \geq 0.5$. Let $\operatorname{TBeta}(a, b ; L, U)$ denote the truncated beta distribution defined on the interval $[L, U]$. Hence, an alternative joint prior for $\left(\theta_{x}, \xi, \eta\right)^{\top}$ to $(5.8)$ is

$$
\begin{aligned}
\theta_{x} & \sim \operatorname{Beta}\left(a_{3}+a_{4}, a_{1}+a_{2}\right), \\
\xi & \sim \operatorname{TBeta}\left(a_{1}, a_{2} ; 0.5,1\right), \quad \text { and } \\
\eta & \sim \operatorname{TBeta}\left(a_{4}, a_{3} ; 0.5,1\right),
\end{aligned}
$$

where $\theta_{x}, \xi$ and $\eta$ are independent. In other words, the joint prior (5.10) is adequate for modeling the assumption of independence between $\xi$ and $\eta$ with restrictions (5.9).

\subsubsection{Joint prior for modeling negative correlation structure}

In some applications, the assumption of independence between $\xi$ and $\eta$ may not be adequate while the negative correlation structure appears to be more practical. One possible way for modeling negative correlation structure is to consider the following inequality constraint:

$$
\xi \geq 1-\eta \text {. }
$$

Define $\xi^{*}=1-\xi, \eta^{*}=1-\eta$. From this inequality constraint, we obtain

$$
\xi^{*} \geq 0, \quad \eta^{*} \geq 0, \quad \text { and } \quad \xi^{*}+\eta^{*} \leq 1 .
$$

Naturally, a Dirichlet prior can be assigned to $\left(\xi^{*}, \eta^{*}\right)^{\top}$. It is well known that the components of a Dirichlet random vector are negatively correlated, and so are $\xi$ and $\eta$.

\subsubsection{Joint prior for modeling positive correlation structure}

Now, we consider the case that $\xi$ and $\eta$ are positively correlated. The first problem is to identify an appropriate prior distribution. The second problem is to compute the corresponding posterior moments for the parameters of interest. Here, we propose a positively correlated bivariate-beta distribution as the joint prior of $(\xi, \eta)$. We then employ the importance sampling to calculate the posterior moments and the DA algorithm to obtain posterior samples. 
5.2.4.1. Positively correlated bivariate-beta distribution. A two-dimensional random vector $\mathbf{w}=\left(w_{1}, w_{2}\right)^{\top}$ is said to follow a positively correlated bivariate-beta distribution, denoted as $\mathbf{w} \sim \operatorname{PCBBeta}\left(\gamma_{1}, \gamma_{2} ; a, b\right)$, if the conditional distributions of $w_{1}$ and $w_{2}$ given $\tau$ are independent, and

$$
w_{i} \mid \tau \sim \operatorname{Beta}\left(\gamma_{i} \tau, \gamma_{i}(1-\tau)\right), \quad \gamma_{i}>0, i=1,2,
$$

where $\tau \sim \operatorname{Beta}(a, b), a>0, b>0$ (see, e.g., Albert and Gupta, 1983; 1985). Theorem 2 below gives the joint density of $\mathbf{w}$ and an algorithm for generating the random vector $\mathbf{w}$.

Theorem 2. If $\mathbf{w}=\left(w_{1}, w_{2}\right)^{\top} \sim \operatorname{PCBBeta}\left(\gamma_{1}, \gamma_{2} ; a, b\right)$, then (i) the density of $\mathbf{w}$ is

$f(\mathbf{w})=\int_{0}^{1} \frac{\tau^{a-1}(1-\tau)^{b-1}}{B(a, b)} \prod_{i=1}^{2} \frac{w_{i}^{\gamma_{i} \tau-1}\left(1-w_{i}\right)^{\gamma_{i}(1-\tau)-1}}{B\left(\gamma_{i} \tau, \gamma_{i}(1-\tau)\right)} d \tau$

(ii) Samples of $\mathbf{w}$ can be generated as follows: First generate a Beta $(a, b)$ random variate $\tau$, and then independently generate $w_{i}$ from $\operatorname{Beta}\left(\gamma_{i} \tau, \gamma_{i}(1-\tau)\right)$ for $i=1,2$.

We obtain the correlation coefficient between $w_{1}$ and $w_{2}$ in the following theorem.

Theorem 3. If $\mathbf{w}=\left(w_{1}, w_{2}\right)^{\top} \sim \operatorname{PCBBeta}\left(\gamma_{1}, \gamma_{2} ; a, b\right)$, then the correlation coefficient between $w_{1}$ and $w_{2}$ is

$$
\rho\left(w_{1}, w_{2}\right)=\sqrt{\frac{\left(\gamma_{1}+1\right)\left(\gamma_{2}+1\right)}{\left(\gamma_{1}+a+b+1\right)\left(\gamma_{2}+a+b+1\right)}} .
$$

The proof of Theorem 3 is given in Appendix B. Theorem 3 shows that the PCBBeta distribution can be used to quantify the positive association between two random variables. In particular, if $\gamma_{1}=\gamma_{2}=\gamma$ and $a=b=1$, then

$$
\rho\left(w_{1}, w_{2}\right)=\frac{\gamma+1}{\gamma+3}=1-\frac{2}{\gamma+3},
$$

which is an increasing function of $\gamma$. The larger the prespecified value of $\gamma$, the stronger the association between $w_{1}$ and $w_{2}$.

5.2.4.2. Computing posterior moments via importance sampling. Substituting (5.7) into (5.1), we can rewrite the likelihood function as

$$
L_{H}\left(\theta_{x}, \xi, \eta \mid Y_{\mathrm{obs}}\right)=\left(1-\theta_{x}\right)^{n_{1}+n_{2}} \cdot h\left(\theta_{x}, \xi, \eta\right),
$$

where

$$
\begin{aligned}
h\left(\theta_{x}, \xi, \eta\right)= & \xi^{n_{1}}\left[p_{2} \xi+(1-\xi)\right]^{n_{2}}\left[p_{3}\left(1-\theta_{x}\right) \xi+\theta_{x}(1-\eta)\right]^{n_{3}} \\
& \cdot\left[p_{4}\left(1-\theta_{x}\right) \xi+\theta_{x} \eta\right]^{n_{4}}
\end{aligned}
$$

Motivated by (5.8) and (5.12), we may consider the following distributions as the joint prior for $\left(\theta_{x}, \xi, \eta\right)^{\top}$ if $\xi$ and $\eta$ are believed to be positively correlated

$$
\begin{aligned}
& \theta_{x} \sim \operatorname{Beta}(\alpha, \beta), \quad(\xi, \eta)^{\top} \sim \operatorname{PCBBeta}(\gamma, \gamma ; 1,1) \\
& \text { and they are independent. }
\end{aligned}
$$

The resultant posterior distribution can be shown to be

$$
\begin{aligned}
f\left(\theta_{x}, \xi, \eta \mid \text { data }\right)= & c_{H}^{-1}(\alpha, \beta, \gamma, \mathbf{n}) \cdot \theta_{x}^{\alpha-1}\left(1-\theta_{x}\right)^{n_{1}+n_{2}+\beta-1} \\
& \cdot f(\xi, \eta) \cdot h\left(\theta_{x}, \xi, \eta\right) .
\end{aligned}
$$

By importance sampling, we obtain

$$
c_{H}(\alpha, \beta, \gamma, \mathbf{n}) \doteq \frac{B\left(\alpha, n_{1}+n_{2}+\beta\right)}{L} \sum_{\ell=1}^{L} h\left(\theta_{x}^{(\ell)}, \xi^{(\ell)}, \eta^{(\ell)}\right),
$$

where $\left\{\theta_{x}^{(\ell)}\right\}_{\ell=1}^{L}$ are a sample of size $L$ from $\operatorname{Beta}\left(\alpha, n_{1}+\right.$ $\left.n_{2}+\beta\right)$ and $\left\{\xi^{(\ell)}, \eta^{(\ell)}\right\}_{\ell=1}^{L}$ are a sample of size $L$ from $\operatorname{PCBBeta}(\gamma, \gamma ; 1,1)$ via Theorem $2(\mathrm{ii})$.

The parameters of interest can be expressed as

$$
\begin{aligned}
\theta_{x} & =\theta_{x}, \quad \theta_{y}=\left(1-\theta_{x}\right)(1-\xi)+\theta_{x} \eta, \quad \text { and } \\
\delta & =\frac{\xi \eta}{(1-\xi)(1-\eta)} .
\end{aligned}
$$

Therefore, the posterior moments of $\theta_{x}$ can be readily calculated via the importance sampling. However, calculations of posterior moments such as $E\left(\theta_{y}^{t} \mid\right.$ data $)$ and $E\left(\delta^{t} \mid\right.$ data $)$ require the evaluation of the expression $\left(\left(1-\theta_{x}\right)(1-\xi)+\theta_{x} \eta\right)^{t}$. $h\left(\theta_{x}, \xi, \eta\right) L$ times at $\left(\theta_{x}^{(\ell)}, \xi^{(\ell)}, \eta^{(\ell)}\right)$ for $\ell=1, \ldots, L$. To this end, the DA algorithm can be adopted for this purpose and we discuss the algorithm as follows.

5.2.4.3. Generating posterior samples via the DA algorithm. The likelihood function (5.14) and the prior assumption (5.15) can be re-formulated in terms of a hierarchical model with three stages. In the first stage, we can augment the observed data $Y_{\text {obs }}$ with three latent variates $\left\{Z_{2}, Z_{3}, Z_{4}\right\}=$ $Y_{\mathrm{mis}}$ so that the complete-data likelihood is given by

$$
\begin{aligned}
L_{H}\left(\theta_{x}, \xi, \eta \mid Y_{\mathrm{obs}}, Y_{\mathrm{mis}}\right)= & \theta_{x}^{Z_{3}+Z_{4}}\left(1-\theta_{x}\right)^{n-Z_{3}-Z_{4}} \\
& \cdot \xi^{Z_{1}}(1-\xi)^{Z_{2}} \cdot \eta^{Z_{4}}(1-\eta)^{Z_{3}}
\end{aligned}
$$

where $Z_{1}=n-Z_{2}-Z_{3}-Z_{4}$. Similar to (5.4), the conditional predictive distribution is

$$
\begin{aligned}
f\left(Y_{\text {mis }} \mid Y_{\text {obs }}, \theta_{x}, \xi, \eta\right) \\
=\operatorname{Binomial~}\left(Z_{2} \mid n_{2},(1-\xi) /\left[p_{2} \xi+1-\xi\right]\right) \\
\quad \cdot \operatorname{Binomial~}\left(Z_{3} \mid n_{3}, \theta_{x}(1-\eta) /\left[p_{3}\left(1-\theta_{x}\right) \xi+\theta_{x}(1-\eta)\right]\right) \\
\quad \cdot \operatorname{Binomial~}\left(Z_{4} \mid n_{4}, \theta_{x} \eta /\left[p_{4}\left(1-\theta_{x}\right) \xi+\theta_{x} \eta\right]\right) .
\end{aligned}
$$

In the second stage, given a hyperparameter $\tau$, the joint prior is a product of independent beta distributions:

$$
\begin{aligned}
f\left(\theta_{x}, \xi, \eta \mid \tau\right)= & f\left(\theta_{x}\right) \cdot f(\xi \mid \tau) \cdot f(\eta \mid \tau) \\
= & \operatorname{Beta}\left(\theta_{x} \mid \alpha, \beta\right) \cdot \operatorname{Beta}(\xi \mid \gamma \tau, \gamma(1-\tau)) \\
& \cdot \operatorname{Beta}(\eta \mid \gamma \tau, \gamma(1-\tau)) .
\end{aligned}
$$

In the third stage, we assign $\tau$ an uniform prior, namely, $f(\tau)=I_{(0,1)}(\tau)$, where $I_{D}(\cdot)$ represents the indicator function of the set $D$. We write the joint distribution of the 
Table 6. Sensitive sexual behavior data

\begin{tabular}{l|ccc|ccc}
\hline \hline \multirow{2}{*}{ Item } & \multicolumn{3}{|c|}{ Self-report $(N=102)$} & \multicolumn{3}{c}{ UCT $(N=244)$} \\
\cline { 2 - 7 }$(N=346)$ & Rate & Yes & No & Rate & Yes & No \\
\hline 1. Sex without a condom & 0.59 & 60 & 42 & 0.70 & 171 & 73 \\
2. Drank until intoxication & 0.77 & 79 & 23 & 0.70 & 171 & 73 \\
3. Sex after drinking & 0.48 & 49 & 53 & 0.49 & 120 & 124 \\
4. Sex without a condom after drinking & 0.36 & 37 & 65 & 0.65 & 159 & 85 \\
5. Had sex & 0.74 & 75 & 27 & 0.84 & 205 & 39 \\
\hline
\end{tabular}

Source: LaBrie \& Earleywine (2000).

complete-data and parameters as $L_{H}\left(\theta_{x}, \xi, \eta \mid Y_{\text {obs }}, Y_{\text {mis }}\right)$. $f\left(\theta_{x}, \xi, \eta \mid \tau\right) \cdot f(\tau)$. Thus, we have

$$
\begin{aligned}
& f\left(\theta_{x}, \xi, \eta \mid Y_{\text {obs }}, Y_{\text {mis }}, \tau\right) \\
& =\operatorname{Beta}\left(\theta_{x} \mid Z_{3}+Z_{4}+\alpha, n-Z_{3}-Z_{4}+\beta\right) \\
& \quad \cdot \operatorname{Beta}\left(\xi \mid Z_{1}+\gamma \tau, Z_{2}+\gamma[1-\tau]\right) \\
& \quad \cdot \operatorname{Beta}\left(\eta \mid Z_{4}+\gamma \tau, Z_{3}+\gamma[1-\tau]\right) \\
& f\left(\tau \mid Y_{\text {obs }}, \xi, \eta\right) \\
& \propto \frac{(\xi \eta)^{\gamma \tau}[(1-\xi)(1-\eta)]^{\gamma(1-\tau)}}{[B(\gamma \tau, \gamma(1-\tau))]^{2}}, \quad \tau \in(0,1) .
\end{aligned}
$$

Sampling from (5.16) and (5.17) is pretty straightforward. Note that (5.18) is an un-normalized one-dimensional density function defined on $(0,1)$. The grid points method (see, e.g., Gelmen et al., 1995, p. 302) can be used to generate random samples from this distribution. The implementation of the Gibbs sampling and the calculation of arbitrary expectations of interest were presented thoroughly in Gelfand \& Smith (1990) and Arnold (1993), and are hence omitted here.

\section{NUMERICAL ILLUSTRATIONS}

\subsection{Sensitive sexual behavior data}

Most studies of sexual behaviors employ conventional self-report surveys. Researchers have long criticized the validity of these self-reports since sexual behavior is often highly private. An alternative approach, called unmatchedcount technique (UCT), provides participants a chance to answer sensitive items without directly admit to the sensitive behavior (Wimbush \& Dalton, 1997). In the UCT method, half of the participants will receive a set of, for instance, five questions (in which all questions are nonsensitive) while the other half will receive a set of six questions (in which one of them is the sensitive question). It should be noted that the five non-sensitive questions are common to all respondents. At the end of the survey, respondents simply indicate the number of statements that are true for them. The base rate estimate for the sensitive item is determined through random assignment of participants and comparisons between the two samples. All samples were obtained via simple random sampling. The main feature of the UCT is that participants do not respond directly to the sensitive item(s).

LaBrie \& Earleywine (2000) used an anonymous selfreport questionnaire and the UCT to estimate the base rates for some sexual risk behaviors (e.g., having sex without a condom and having sex without a condom after drinking). Three hundred forty-six college students were randomly divided into three groups. Group 1 (102 subjects) received a true/false conventional self-report survey. Groups 2 (122 subjects) and 3 (122 subjects) were UCT protocol groups, with Group 2 receiving Form A and Group 3 receiving Form B (see Appendix B in LaBrie \& Earleywine, 2000, for more details). Their findings are reported in Table 6. For example, $36 \%$ of the respondents receiving the conventional survey endorsed having sex without a condom after consuming alcohol while the UCT protocol revealed a base rate estimate of $65 \%$ for the same behavior. Thus, the anonymous self-report questionnaire revealed only half the percentage of persons engaging in risky sexual behavior after drinking reported by the UCT protocol.

To illustrate the proposed methods in Section 3, for the third sensitive item, we combine the numbers of "Yes" and "No" with those for the two survey methods, resulting in $n=346, n_{\text {yes }}=49+120=169$ and $n_{\text {no }}=$ $53+124=177$. In the triangular model, we further let $\pi=\operatorname{Pr}(Y=1)=\operatorname{Pr}$ (having sex after drinking) and $p=$ $\operatorname{Pr}(W=1)=0.5$. For the ideal situation (i.e., no sampling errors), the observed counts in the triangle would be $s=n_{\text {no }} / 2+n_{\text {yes }} \approx 258$. Therefore, we obtain the observed data $Y_{\mathrm{obs}}=\{n, s, n-s\}=\{346,258,88\}$. Using (3.3), we have $E\left(\pi \mid Y_{\text {obs }}\right)=0.488506$ and $E\left(\pi^{2} \mid Y_{\text {obs }}\right)=0.240819$. Thus, the $95 \%$ Bayesian credible interval (CI) for $\pi$ based on normality approximation is given by $[0.396960,0.580052]$.

Using $\pi^{(0)}=0.5$ as an initial value, the EM algorithm (3.6) and (3.7) converges in 28 iterations. The resultant posterior mode of $\pi$ is $\tilde{\pi}_{T}=0.49133$, which is very close to the Bayesian mean $E\left(\pi \mid Y_{\text {obs }}\right)$. Using the exact IBF sampling described in Section 3.3, we generate $L=20,000$ i.i.d. posterior samples from $f\left(\pi \mid Y_{\text {obs }}\right)$. The histogram based on these samples is plotted in Figure 2(b), which shows that the exact IBF sampling can recover the density completely. The corresponding posterior mean, standard error and $95 \%$ Bayesian CI for $\pi$ are $0.488805,0.0468918$ and [0.395190, 0.577961]. 
(a)

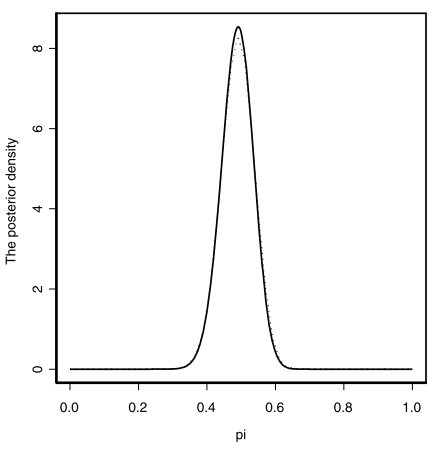

(b)

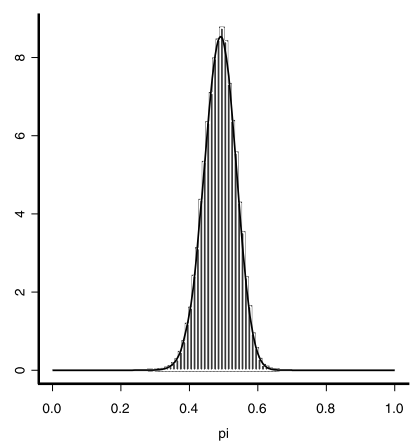

Figure 2. Posterior distribution of $\pi=\operatorname{Pr}($ having sex after drinking) under the uniform prior (i.e., $a=b=1$ ) and $p=\operatorname{Pr}(W=1)=0.5$ for the non-randomized triangular model for $n=346$ and $s=258$. (a) The comparison between the posterior distribution (solid curve) exactly given by (3.1) with the dotted curve estimated by a kernel density smoother based on i.i.d. posterior samples generated via the exact IBF sampling. (b) The histogram based on i.i.d. posterior samples generated via the exact IBF sampling.

\subsection{Induced abortion data}

Liu \& Chow (1976) considered an induced abortion study in Taichung City and Taoyuan County, Taiwan (see also Winkler and Franklin, 1979). They adopted the multipletrial version of the Warner model to increase the efficiency of estimation. Since the present paper only discusses the single-trial Warner model with the crosswise model as its non-randomized version, we simply use the data from the first trial of each respondent. The target population of interest in this study is those married women of age 20 to 44 in the South District of Taichung City, Taiwan. The investigators would like to estimate the incidence rate of induced abortions in the target population. With $p=0.3$, the survey yielded 90 "Yes" answers (i.e., $r=\sum_{i=1}^{n} y_{i}^{\mathrm{HW}}=90$ in (4.1)) and 60 "No" answers (i.e., $n=150$ ). Using likelihoodbased method, the proportion of married women of childbearing age who have had induced abortion is estimated to be $\hat{\pi}_{W}=0.25$ with estimated variance being $\widehat{\operatorname{Var}}\left(\hat{\pi}_{W}\right)=0.01$ (Migon \& Tachibana, 1997, p. 406). The resultant 95\% CI of $\pi$ is $[0.25-1.96 \sqrt{0.01}, 0.25+1.96 \sqrt{0.01}]=[0.054,0.446]$.

To illustrate the proposed methods in Section 4, we consider the uniform prior (i.e., $a=b=1$ ). Note that $p=0.3$ and the observed data $Y_{\mathrm{obs}}=\{n, r, n-r\}=$ $\{150,90,60\}$. Using (4.2), we obtain $E\left(\pi \mid Y_{\text {obs }}\right)=0.2544$ and $E\left(\pi^{2} \mid Y_{\text {obs }}\right)=0.0742$. Thus, $\operatorname{Var}\left(\pi \mid Y_{\text {obs }}\right)=0.0095$ so that the $95 \%$ Bayesian CI for $\pi$ based on normality approximation is $[0.0632,0.4457]$.

Using $\pi^{(0)}=0.5$ as an initial value, the EM algorithm (4.5) and (4.6) converges in 96 iterations. The posterior mode of $\pi$ is $\tilde{\pi}_{W}=0.25$, which is same as the MLE $\hat{\pi}_{W}$. (a)
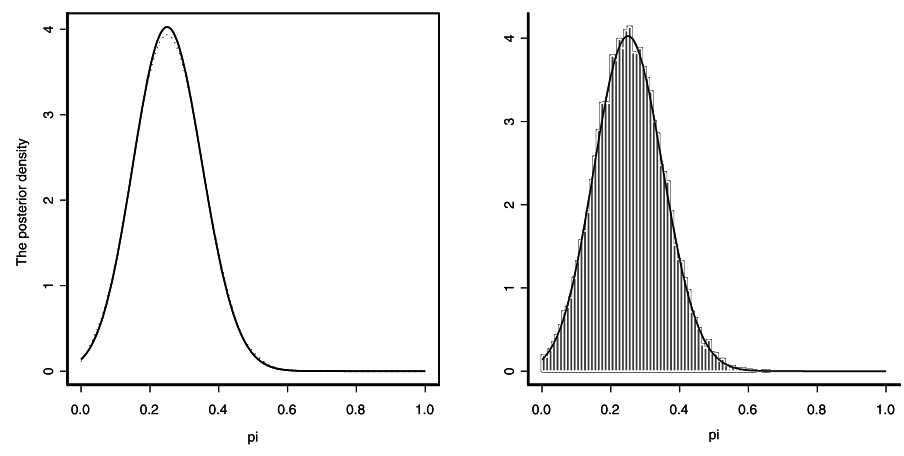

Figure 3. Posterior distribution of

$\pi=\operatorname{Pr}($ having induced abortion) under the uniform prior

(i.e., $a=b=1$ ) and $p=\operatorname{Pr}(W=1)=0.3$ for the

non-randomized cross model for $n=150$ and $r=90$. (a) The comparison between the posterior distribution (solid curve) exactly given by (4.1) with the dotted curve estimated by a kernel density smoother based on i.i.d. posterior samples generated via the exact IBF sampling. (b) The histogram based on i.i.d. posterior samples generated via the exact IBF sampling.

Using the exact IBF sampling described in Section 4.3, we generate $L=20,000$ i.i.d. posterior samples from $f\left(\pi \mid Y_{\text {obs }}\right)$. The histogram based on these samples is plotted in Figure $3(\mathrm{~b})$, which shows that the exact IBF sampling recovers the density completely. The corresponding posterior mean, standard error and 95\% Bayesian CI for $\pi$ are 0.2546, 0.0973 and $[0.0680,0.4490]$.

\subsection{HIV data}

Strauss et al. (2001) reported an HIV data set which examined the relationship between self-reported HIV status and history of sex exchange for drugs and money. All participants were drug dependent women offenders who were mandated to treatment through the criminal justice system of New York City. The data were collected as part of an evaluation study of four drug treatment programs, respectively classified as prison-based, jailed-based, community-based residential and community-based outpatient. The data reflected baseline responses from 325 clients interviewed at the four treatment programs from May, 1995 through December, 1996. Notice that there are incomplete data for 83 subjects. Table 7 gives the cross-classification of history of sex exchange (no or yes, denoted by $X=0$ or $X=1$ ) and HIV status (negative or positive, denoted by $Y=0$ or $Y=1)$ as reported by the women. The objective is to examine if association exists between sex exchange and HIV status. Obviously, both questions (i.e., sex history and HIV status) are highly sensitive questions to respondents.

To illustrate the proposed methods in Section 5.1, we let $W=i$ if the respondent was born in the $k$-th quarter, and it 
Table 7. HIV data from Strauss et al. (2001)

\begin{tabular}{l|ll|c}
\hline \hline History of & \multicolumn{3}{|c}{ HIV status } \\
\cline { 2 - 4 } sex exchange & $Y=0(\mathrm{HIV}-)$ & $Y=1(\mathrm{HIV}+)$ & missing \\
\hline$X=0$ (no) & $108\left(m_{1}, \theta_{1}\right)$ & $18\left(m_{2}, \theta_{2}\right)$ & 44 \\
$X=1$ (yes) & $93\left(m_{3}, \theta_{3}\right)$ & $23\left(m_{4}, \theta_{4}\right)$ & 39 \\
\hline Note: $X$ denotes history of sex exchange and $Y$ denotes HIV status.
\end{tabular}

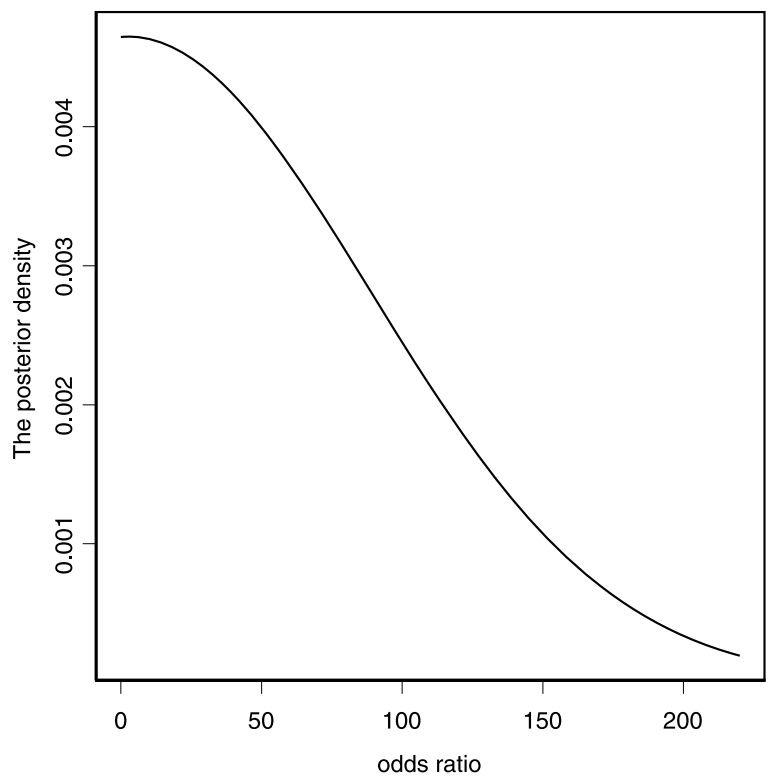

Figure 4. The posterior density of the odds ratio $\delta$ estimated by a kernel density smoother based on the last 20,000 posterior samples generated by the DA algorithm.

is thus reasonable to assume that $p_{i}=\operatorname{Pr}\{W=i\}=0.25$ for $i=1, \ldots, 4$, and $W$ is independent of the two sensitive questions. For the ideal situation (i.e., no sampling errors), the observed counts would be $n_{1}=m_{1} / 4=27, n_{2}=27+m_{2}=$ $45, n_{3}=27+m_{3}=120$ and $n_{4}=27+m_{4}=50$ if the missing data in Table 7 are ignored. To consider the situation with sampling errors, we first generate 50 i.i.d. samples from Multinomial $\left(108 ;(0.25, \ldots, 0.25)^{\top}\right)$, then calculate the average of these counts, and finally yield $\left(n_{1}^{\prime}, \ldots, n_{4}^{\prime}\right)=$ $(28,26,26,28)^{\top}$. Therefore, we obtain the observed frequencies $Y_{\text {obs }}=\left\{n ; n_{1}, \ldots, n_{4}\right\}=\{242,28,44,119,51\}$.

Using $\theta^{(0)}=(0.25,0.25,0.25,0.25)^{\top}$ as initial values, the EM algorithm (5.5) and (5.6) converges in 100 iterations.
The posterior modes of $\boldsymbol{\theta}$ and odds ratio $\delta$ are listed in the second column of Table 8. Based on (5.3) and (5.4), we employ the DA algorithm to generate 40,000 posterior samples and only use the second half of the samples. The Bayes estimates of $\boldsymbol{\theta}$ and $\delta$ are given in Table 8. Since the Bayes CIs include the value of 1 , we have reason to believe that there is no association between sex exchange and HIV status. Figure 4 shows the posterior density of the odds ratio $\delta$ estimated by a kernel density smoother based on the last 20,000 posterior samples generated by the DA algorithm.

\section{DISCUSSION}

Yu et al. (2008) and Tian et al. (2007) studied the survey designs for the triangular, crosswise and hidden sensitivity models, respectively. They investigated these models from a frequentist perspective. In this article, we on the other hand focus on the analysis of these models in the Bayesian framework. The Bayesian framework provides a natural way to study these models when only partial information are available. Furthermore, the Bayesian approach is particularly appealing when sample information are relatively limited.

For the HS model, the resultant MLE $\hat{\theta}=\left(\hat{\theta}_{1}, \ldots, \hat{\theta}_{4}\right)^{\top}$ with explicit form is possibly invalid, i.e., it does not satisfy: $\hat{\theta}_{i} \geq 0, i=1, \ldots, 4, \sum_{i=1}^{4} \hat{\theta}_{i}=1$. However, we do not have this issue in the Bayesian framework.

In practice, the selection of prior is very important to researchers. For the HS model, the choice of a Dirichlet prior is equivalent to a product of three independent beta distributions (see (5.8)). In some cases, the assumption of independence between $\xi$ and $\eta$ may not be appropriate. For modeling independence with restrictions and negative correlation structure, we suggest two distinct priors (see (5.10) and (5.11)). By implementing a very simple sampling algorithm, the PCBBeta distribution becomes desirable for modeling positive correlation structure. The control of similarity can be fine tuned by selecting appropriate value of the parameter $\gamma$. Based on the PCBBeta prior, we employ the importance sampling to calculate the corresponding posterior moments.

The DA algorithm is a special Gibbs sampler. In order to implement the DA algorithm, we first formulate the original problem into a hierarchical models with three stages. We then derived the full conditional distributions (5.16)-(5.18) with simple sampling methods.

Table 8. Posterior modes and estimates of parameters for HIV data

\begin{tabular}{l|c|c|c|c}
\hline \hline Parameters & Posterior mode & Bayes mean & Bayes std & $95 \%$ Bayes CI \\
\hline$\theta_{1}$ & 0.4628 & 0.4635 & 0.0757 & {$[0.3208,0.6172]$} \\
$\theta_{2}$ & 0.0661 & 0.0680 & 0.0317 & {$[0.0105,0.1339]$} \\
$\theta_{3}$ & 0.3760 & 0.3723 & 0.0426 & {$[0.2877,0.4538]$} \\
$\theta_{4}$ & 0.0950 & 0.0960 & 0.0346 & {$[0.0300,0.1648]$} \\
$\delta=\theta_{1} \theta_{4} / \theta_{2} \theta_{3}$ & 1.7692 & 3.6939 & 31.431 & {$[0.5733,11.932]$} \\
\hline
\end{tabular}




\section{APPENDIX A. THE EXACT IBF SAMPLING}

Suppose that both the complete-data posterior distribution $f\left(\pi \mid Y_{\mathrm{obs}}, Z\right)$ and the conditional predictive distribution $f\left(Z \mid Y_{\mathrm{obs}}, \pi\right)$ are available. The fundamental conditional sampling principle states that: If we could obtain independent samples $\left\{Z^{(\ell)}\right\}_{\ell=1}^{L}$ from $f\left(Z \mid Y_{\text {obs }}\right)$ and generate $\pi^{(\ell)} \sim f\left(\pi \mid Y_{\mathrm{obs}}, Z^{(\ell)}\right)$ for $\ell=1, \ldots, L$, then $\left\{\pi^{(\ell)}\right\}_{1}^{L}$ are i.i.d. samples from the observed posterior distribution $f\left(\pi \mid Y_{\text {obs }}\right)$. In other words, the key issue is to generate independent samples from $f\left(Z \mid Y_{\text {obs }}\right)$.

Let $\mathcal{S}_{\left(\pi \mid Y_{\text {obs }}\right)}$ and $\mathcal{S}_{\left(Z \mid Y_{\text {obs }}\right)}$ denote the conditional supports of $\pi \mid Y_{\text {obs }}$ and $Z \mid Y_{\text {obs }}$, respectively. The sampling-wise IBF states that (Tan et al., 2003)

$f\left(Z \mid Y_{\mathrm{obs}}\right) \propto \frac{f\left(Z \mid Y_{\mathrm{obs}}, \pi_{0}\right)}{f\left(\pi_{0} \mid Y_{\mathrm{obs}}, Z\right)}, \begin{aligned} & \text { for any arbitrary } \pi_{0} \in \mathcal{S}\left(\pi \mid Y_{\mathrm{obs}}\right) \\ & \text { and all } Z \in \mathcal{S}\left(Z \mid Y_{\mathrm{obs}}\right) .\end{aligned}$

When $Z$ is a discrete random variable/vector taking finite values on the domain, we denote the conditional support of $Z \mid\left(Y_{\mathrm{obs}}, \pi\right)$ by $\mathcal{S}_{\left(Z \mid Y_{\mathrm{obs}}, \pi\right)}=\left\{z_{1}, \ldots, z_{K}\right\}$. Since $f\left(Z \mid Y_{\mathrm{obs}}, \pi\right)$ is available, we can first directly identify $\left\{z_{k}\right\}_{1}^{K}$ from the model specification and all $\left\{z_{k}\right\}_{1}^{K}$ become known. Noting that $\left\{z_{k}\right\}_{1}^{K}$ generally do not depend on $\pi$, we have $\mathcal{S}_{\left(Z \mid Y_{\mathrm{obs}}\right)}=\mathcal{S}_{\left(Z \mid Y_{\mathrm{obs}}, \pi\right)}=\left\{z_{1}, \ldots, z_{K}\right\}$. Due to the discreteness of $Z$, the notation $f\left(z_{k} \mid Y_{\text {obs }}\right)$ will be used to denote the probability mass function, i.e., $f\left(z_{k} \mid Y_{\text {obs }}\right)=\operatorname{Pr}\{Z=$ $\left.z_{k} \mid Y_{\mathrm{obs}}\right\}$. Therefore, it suffices to find $\omega_{k}=f\left(z_{k} \mid Y_{\mathrm{obs}}\right)$ for $k=1, \ldots, K$. For any $\pi_{0} \in \mathcal{S}\left(\pi \mid Y_{\text {obs }}\right)$, let

(A.2)

$q_{k}\left(\pi_{0}\right)=\operatorname{Pr}\left\{Z=z_{k} \mid Y_{\mathrm{obs}}, \pi_{0}\right\} / f\left(\pi_{0} \mid Y_{\mathrm{obs}}, z_{k}\right), \quad k=1, \ldots, K$.

From the sampling-wise IBF (A.1), we immediately obtain

$$
\omega_{k}=q_{k}\left(\pi_{0}\right) / \Sigma_{k^{\prime}=1}^{K} q_{k^{\prime}}\left(\pi_{0}\right), \quad k=1, \ldots, K
$$

and $\left\{\omega_{k}\right\}_{1}^{K}$ are independent of $\pi_{0}$. Thus, it is easy to sample from $f\left(Z \mid Y_{\text {obs }}\right)$ since it is a discrete distribution with probability $\omega_{k}$ on $z_{k}$ for $k=1, \ldots, K$. We summarize the algorithm as follows (Tian, Tan \& Ng, 2007).

THE EXACT IBF SAMPLING:

(i) Identify $\mathcal{S}_{\left(Z \mid Y_{\text {obs }}\right)}=\mathcal{S}_{\left(Z \mid Y_{\text {obs }}, \pi\right)}=\left\{z_{1}, \ldots, z_{K}\right\}$ from $f\left(Z \mid Y_{\text {obs }}, \pi\right)$ and calculate $\left\{\omega_{k}\right\}_{1}^{K}$ according to (A.3) and (A.2);

(ii) Generate i.i.d. samples $\left\{Z^{(\ell)}\right\}_{\ell=1}^{L}$ of $Z$ from the probability mass function $f\left(Z \mid Y_{\text {obs }}\right)$ with probabilities $\left\{\omega_{k}\right\}_{1}^{K}$ on $\left\{z_{k}\right\}_{1}^{K}$

(iii) Generate $\pi^{(\ell)} \sim f\left(\pi \mid Y_{\text {obs }}, Z^{(\ell)}\right)$ for $\ell=1, \ldots, L$, then $\left\{\pi^{(\ell)}\right\}_{1}^{L}$ are i.i.d. samples from the observed posterior distribution $f\left(\pi \mid Y_{\text {obs }}\right)$.

\section{APPENDIX B. THE PROOF OF THEOREM 3}

Let $\mu$ and $\sigma^{2}$ denote the mean and variance of $\tau$, respectively. We have $\mu=a /(a+b)$ and $\sigma^{2}=a b /\{(a+b+1)(a+$ $\left.b)^{2}\right\}$. Using the rule of conditional expectation, we obtain

$$
\begin{aligned}
E\left(w_{i}\right) & =E\left\{E\left(w_{i} \mid \tau\right)\right\}=E(\tau)=\mu, \quad i=1,2, \\
E\left(w_{i}^{2}\right) & =E\left\{E\left(w_{i}^{2} \mid \tau\right)\right\}=E\left(\frac{\gamma_{i} \tau^{2}+\tau}{\gamma_{i}+1}\right) \\
& =\frac{\gamma_{i}\left(\sigma^{2}+\mu^{2}\right)+\mu}{\gamma_{i}+1}, \quad i=1,2, \\
E\left(w_{1} w_{2}\right) & =E\left\{E\left(w_{1} w_{2} \mid \tau\right)\right\}=E\left(\tau^{2}\right)=\sigma^{2}+\mu^{2}, \\
\operatorname{Var}\left(w_{i}\right) & =\frac{\gamma_{i} \sigma^{2}+\mu-\mu^{2}}{\gamma_{i}+1}, \quad i=1,2 .
\end{aligned}
$$

Hence,

$$
\begin{aligned}
\rho\left(w_{1}, w_{2}\right) & =\frac{E\left(w_{1} w_{2}\right)-E\left(w_{1}\right) E\left(w_{2}\right)}{\sqrt{\operatorname{Var}\left(w_{1}\right) \cdot \operatorname{Var}\left(w_{2}\right)}} \\
& =\sqrt{\frac{\left(\gamma_{1}+1\right)\left(\gamma_{2}+1\right)}{\left(\gamma_{1}+\left(\mu-\mu^{2}\right) / \sigma^{2}\right)\left(\gamma_{2}+\left(\mu-\mu^{2}\right) / \sigma^{2}\right)}} .
\end{aligned}
$$

Noting that $\left(\mu-\mu^{2}\right) / \sigma^{2}=a+b+1$, we obtain (5.13) immediately.

\section{ACKNOWLEDGEMENTS}

M.-L. Tang's research was fully supported by a grant from the Research Grant Council of the Hong Kong Special Administrative Region (Project Nos. HKBU261508).

\section{Received 28 January 2008}

\section{REFERENCES}

Albert, J. H. and Gupta, A. K. (1983). Estimation in contingency tables using prior information. Journal of the Royal Statistical Society, Series B 45 60-69. MR0701077

Albert, J. H. and Gupta, A. K. (1985). Bayesian methods for binomial data with applications to a nonresponse problem. Journal of the American Statistical Association 80 167-174. MR0786603

Arnold, S. F. (1993). Gibbs sampling. In Handbook of Statistics: Computational Statistics (C. R. Rao, ed.) Vol. 9 599-625. Elsevier Science Publishers B.V., New York. MR1245275

Bar-Lev, S. K., Bobovich, E., and Boukai, B. (2003). A common conjugate prior structure for several randomized response models. Test 12(1) 101-113. MR1993288

Chang, H. J. and LiAng, D. H. (1996). A two-stage unrelated randomized response procedure. Austral. J. Statist. 38 43-51. MR1410801

Dempster, A. P., Laird, N. M., and Rubin, D. B. (1977). Maximum likelihood estimation from incomplete data via the EM algorithm (with discussion). Journal of the Royal Statistical Society, Series B 39 1-38. MR0501537

DiPietro, M. (2004). Bayesian randomized response as a class project. The American Statistician 58(4) 303-309. MR2109420

Gelfand, A. E. and Smith, A. F. M. (1990). Sampling-based approaches to calculating marginal densities. Journal of the American Statistical Association 85 398-409. MR1141740

Gelman, A., Carlin, J. B., Stern, H. S., and Rubin, D. B. (1995). Bayesian Data Analysis. Chapman \& Hall, London. MR1385925

Gjestvang, C. R. and Singh, S. (2006). A new randomized response model. Journal of the Royal Statistical Society, Series B 68 523530. MR2278339 
Greenberg, B. G., Abul-Ela, A. A., Simmons, W. R., and Horvitz, D. G. (1969). The unrelated question randomized response model: theoretical framework. Journal of the American Statistical Association 64 520-539. MR0247719

Horvitz, D. G., Shah, B. V., and Simmons, W. R. (1967). The unrelated question randomized response model. In 1967 Proceedings of the Social Statistics Section, American Statistical Association $65-72$.

Kim, J. M., Tebbs, J. M., and AN, S. W. (2006). Extensions of Mangat's randomized-response model. Journal of Statistical Planning and Inference 136 1554-1567. MR2253781

KUK, A. Y. C. (1990). Asking sensitive questions indirectly. Biometrika 77 436-438. MR1064822

LaBrie, J. W. and Earleywine, M. (2000). Sexual risk behaviors and alcohol: Higher base rates revealed using the unmatched-count technique. The Journal Sex Research 37(4) 321-326.

LiU, P. T. and CHOw, L. P. (1976). The efficiency of the multiple trial randomized response technique. Biometrics 32 607-618.

Mangat, N. S. (1994). An improved randomized response strategy. Journal of the Royal Statistical Society, Series B 56 93-95. MR1257798

MANGAT, N. S. and Singh, R. (1990). An alternative randomized response procedure. Biometrika 77 439-442. MR1064823

Migon, H. S. and TaChibana, V. M. (1997). Bayesian approximations in randomized response model. Computational Statistics and Data Analysis 24 401-409. MR1457917

O'HAGAN, A. (1987). Bayes linear estimators for randomized response models. Journal of the American Statistical Association 82580 585. MR0898362

Pitz, G. F. (1980). Bayes analysis of random response models. Psychological Bulletin 87 209-212.

Spurrier, J. D. and PAdGetT, W. J. (1980). The application of Bayesian techniques in randomized response. Sociological Methodology 11 533-544.

Strauss, S. M., Rindskopf, D. M., and Falkin, G. P. (2001). Modeling relationships between two categorical variables when data are missing: Examining consequences of the missing data mechanism in an HIV data set. Multivariate Behavioral Research 36(4) 471-500.

TAN, M., Tian, G. L., and NG, K. W. (2003). A non-iterative sampling method for computing posteriors in the structure of EM-type algorithms. Statistica Sinica 13(3) 625-639. MR1997165

TANner, M. A. and Wong, W. H. (1987). The calculation of posterior distributions by data augmentation (with discussion). Journal of the American Statistical Association 82 528-540. MR0898357

Tian, G. L., TAN, M., and NG, K. W. (2007). An exact non-iterative sampling procedure for discrete missing data problems. Statistica Neerlandica 61(2) 232-242. MR2323118

Tian, G. L., Yu, J. W., Tang, M. L., and Geng, Z. (2007). A new non-randomized model for analyzing sensitive questions with binary outcomes. Statistics in Medicine 26(23) 4238-4252.

UnNikRisknan, N. K. and Kunte, S. (1999). Bayesian analysis for randomized response models.Sankhyā, Series B 61 422-432.

\section{MR1745745}

WARNER, S. L. (1965). Randomized response: a survey technique for eliminating evasive answer bias. Journal of the American Statistical Association 60 63-69.

Wimbush, J. C. and Dalton, D. R. (1997). Base rate for employee theft: Convergence of multiple methods. Journal of Applied Psychology 82 756-763.

Winkler, R. L. and Franklin, L. A. (1979). Warner's randomized response model: a Bayesian approach. Journal of the American Statistical Association 74 207-214. MR0529538

Yu, J. W., Tian, G. L., and TANG, M. L. (2008). Two new models for survey sampling with sensitive characteristic: Design and analysis. Metrika 67 251-263.

Zou, G. H. (1997). Two-stage randomized response procedures as single stage procedures. Austral. J. Statist. 39 235-236. MR1481265

\section{Guo-Liang Tian}

Department of Statistics and Actuarial Science

The University of Hong Kong

Pokfulam Road, Hong Kong

P. R. China

E-mail address: gltian@hku.hk

Division of Biostatistics

University of Maryland Greenebaum Cancer Center

MSTF Suite 261, 10 South Pine Street

Baltimore, Maryland 21201

U.S.A.

Kam Chuen Yuen

Department of Statistics and Actuarial Science

The University of Hong Kong

Pokfulam Road, Hong Kong

P. R. China

Man-Lai Tang

Department of Mathematics

Hong Kong Baptist University

Kowloon Tong, Hong Kong

P. R. China

Ming T. Tan

Division of Biostatistics

University of Maryland Greenebaum Cancer Center

MSTF Suite 261, 10 South Pine Street

Baltimore, Maryland 21201

U.S.A. 